\title{
ARTICLE
}

\section{Out of the Depths: Engineering, Submersibles and Assemblages}

\author{
John Reader \\ William Temple Foundation and University of Chester, UK, drjohnreader@hotmail.co.uk
}

Environmental concerns about the state of the world's oceans have been growing over recent years, particularly as acidification, overfishing and the limited capacity of the oceans to absorb $\mathrm{CO}^{2}$ from climate change have come to the fore. Engineering practices and innovations in a number of forms are of direct relevance to this, notably through a concern to develop engineering in such a way as to be for the benefit of all, including the non-human world. This article argues that assemblage theory offers an alternative way of understanding how culture is always already a part of nature, and that human autonomy has to be seen as constrained and limited if the worst effects of pollution and climate change are to be addressed.

KEYWORDS: assemblage theory, carbon capture and storage (CCS), marine biology, New Materialism

This article will examine current developments from within marine biology, assemblage theory and engineering, not necessarily an obvious combination of disciplines, but one that will aim to illuminate how collectively they can address some of the more serious environmental challenges that are now to be faced. It will be established that this forms a legitimate concern for engineering; that what happens in the oceans is of considerable importance for the future of the planet, and then that the new approach represented by this particular philosophical concept provides exactly the linking between the disciplines that is required. Once this territory has been laid out, the aim is to examine some specific areas in greater detail in order to consolidate the argument.

In a recent book on engineering as a means to working towards greater social justice, the authors suggested that the critical reflection required of engineers in this process involves asking three sets of related questions: who are we working for and whose needs are we serving?; how is the product or service created and is this being done in an equitable way?; and, who benefits socially and economically from the work being done? (Baillie, Kabo, \& Reader, 2013). What is being encouraged is the creation of time and space for reflection and the willingness to enter into the liminal space or heterotopia that will enable appropriate courses of action to emerge (Baillie et al., 2013, pp. 90-91). There is no doubt that attempting to ensure that engineering innovations and practices are for the benefit of the whole of society must include concerns for environmental issues and ways in which both are developed either to improve the current state of the planet or to damage prospects for the future. Referring also to the work of Bruno Latour, one of the originators of assemblage theory, the authors emphasize that all decisions must be judged in the light of the specific context, with the understanding that none are neutral or value free, but that all contain values from the beginning, however implicit those might be. Thus one of the tasks for engineers is to identify the particular values that are always already embedded in the process, and to be prepared both to challenge those 
and to propose alternatives (Baillie et al., 2013, p. 72). It will be argued that this entails a mapping that must go beyond the purely technological or scientific and that takes the discussions into the realms of philosophy, politics and ethics.

\section{MARINE BIOLOGY}

So why are the oceans so important for the flourishing of life, both human and non-human? As one of the accessible introductions to the subject of marine biology argues, we are beginning to appreciate that the marine environment and the organisms that live within it are critical to our wellbeing and survival (Mladenov, 2013). Thus, amongst other things, it provides us and our livestock with rich sources of food; it helps to stabilize the climate, which is critical as the impacts of human-induced climate change become ever more evident; it absorbs many of our waste products; it provides us with a wide range of biomolecules which are important in medicine and engineering; coral reefs and mangrove systems protect coastlines from erosion, and marine ecosystems support recreation and tourism throughout the world. Each of these is now under threat as human activities such as overfishing, coastal development, sewage disposal, plastic pollution, oil spills and the increased emissions of greenhouse gases cause significant damage to the marine environment and the life forms living in it. The pressures coming from the projected increase of the human population from seven to nine billion over the next forty years will intensify the dangers to this crucial source of life on the planet.

To add a little more detail to this: there is a growing scientific consensus that increasing global average air temperatures brought about by climate change are resulting in increasing global ocean temperatures. The average temperature of the world's oceans is one degree centigrade higher than 140 years ago and is predicted to continue to rise during this century. This warming is causing a rapid decrease in the thickness and coverage of sea ice in the Arctic Ocean bringing its own dangers as sea levels rise and areas of human habitation come under threat. What is often less recognized is that the oceans are a vast reservoir of planetary carbon and that the world's oceans are an enormous natural sink for atmospheric carbon dioxide. Currently they absorb about $25 \%$ of the roughly 35 billion tons of carbon dioxide emitted into the atmosphere each year by humans burning fossil fuels and deforesting the planet's surface. Combined, the oceans and the forests account for about $50 \%$ of $\mathrm{CO}^{2}$ being absorbed but this means that even now there is a 2 parts per million annual rise in the levels in the atmosphere and the pre-industrial level of $278 \mathrm{ppm}$ is now greater than $380 \mathrm{ppm}$ and rising. Although there is much debate and controversy about what might be "safe" levels of this concentration, it could be that these have already been exceeded. One of the concerning impacts of this is ever greater acidification of the oceans themselves, with estimates that they are now 30\% more acidic than in the pre-industrial era. Not only does this have a profound effect upon marine organisms and the overall functioning of the marine ecosystem, but it means that the capacity of the oceans to absorb increasing levels of $\mathrm{CO}^{2}$ is becoming more limited, thus threatening unpredictable feedback systems in the overall climate. One possible impact is upon what is known as the Great Ocean Conveyor which currently moderates the global climate by distributing oxygen, nutrients and heat around the globe, but which may be adversely impacted by the ice melt and result in a drastic cooling of the European climate (Mladenov, 2013, p. 18). It does not take a great deal of imagination to work out that what happens to the world's oceans is of great importance for the wellbeing of the planet and human society as a whole.

It will be shown in due course how engineering has a direct relevance to and impact upon these environmental concerns, but it now needs to be established how and why the philosophical ideas referred to earlier play a crucial role in this process. One of the major debates that has arisen as a result of the environmental crisis is that of how humans understand themselves in relation to what 
might be called "the Natural World". It has been suggested that the problems humans have created are the result of seeing ourselves as lone autonomous individuals relating to the rest of nature only as if it is a resource to be used and exploited for our own instrumental purposes. This leads to the misguided view that such resources are either without limit, or can be replaced by substitutes created by human technology and ingenuity. Thus if one source of energy is about to run out or to become increasingly difficult or expensive to access-hence for instance the discussions about Peak Oil-we simply develop the alternative technologies to replace it with renewable sources of energy such as wind power or wave power. Whilst this is not to deny the importance of responding in this way, it begs the underlying question of whether, as humans, we have failed to acknowledge the ways in which we are not simply external manipulators of an inanimate resource, but are in fact always already part of the ecosystems which both shape us and are shaped by our technological culture. Thus we are challenged to develop new understandings of what it is to be human, but now in relation to all those non-humans, both other living creatures, but also the machines and technological devices which we like to think are our creations and which we imagine we control. The philosophical sources to be drawn on in this article pursue a critique of this limiting and damaging concept of human autonomy and suggest that what is required is an alternative discourse and conceptual framework which uses the notion of assemblages-an assemblage being a particular combination of the human and non-human functioning in a specific context, and within which the role of the human is more constrained and far less of a controlling factor.

\section{ASSEMBLAGES}

Up to this point in the argument the focus has been upon practical examples from marine biology and the environmental issues which are at stake. One has used the terminology of assemblages within this context in order to provide a general view of what is actually a quite technical and specialized development within philosophy, arguing that there are important ethical consequences of employing the term in this way. At one level this is a device for mapping the complex interconnections that exist between the human and the non-human and between different disciplines as they inform and shape what has been described as culture in its full diversity, but which I suggest is better seen as another aspect of the nature of which we are a part. It also proposes an alternative understanding of what it is to be human which challenges the established interpretation of individual autonomy and control, and which acknowledges that the human itself is always in the process of change and development and not to be reduced to some essentialist understanding. Each of these aspects of the theory is capable of much greater exposition than is possible in a short article, but it is necessary to offer some pointers to the thinkers involved, their basic ideas, and how this enables the sort of ethical judgements it is necessary to make. It needs to be recognized that not all of the theorists involved use the term in the same way, but that is less important than how it can be of value for the particular concerns articulated here.

A major interpretation of assemblages is that they provide a way of describing the entangled and complex nature of the relationships and connections which go to make up very specific contexts or situations that humans encounter, and of which we are always already a part. Thus one of the deepseated problems that humans have created, is that we have differentiated our views of the world into a series of specialized disciplines and discourses, which serve now to produce division, misunderstanding and general lack of communication. One way of describing this is that we operate in silos, unable to cross apparent boundaries into other modes of thought or existence. This is a massively disabling factor as we struggle to address the very problems that have been created by the independent development of each of these areas, but without reference to each other, let alone to the insights and theories available from each discipline. To assume that this is simply an academic problem would be a mistake. In politics, for instance, one encounters a high degree of 
disconnection, and the disillusion of many voters from the views and ways of operating of professional politicians. "They" exist in a small bubble surrounded by press officers and the media with whom they mix on a daily basis, apparently representing the vested interests of the elite groups from which they come, and making decisions about "our" lives without any direct experience of or reference to the realities which "we" face. How often does one hear this view expressed in our heavily compartmentalized culture? Yet political and commercial factors are often a crucial part of the mix - or assemblage-which constitute those matters of concern of which we should all be aware. In this way, the discourse of assemblages is a means of putting back together, or reassembling, the actual relationships and connections that determine or shape the lives we are leading.

This is not simply a matter, however, of reconnecting human relationships across various boundaries, although some of the theorists would not go further than this, but also of acknowledging the material world, the elements which we define as inanimate, but also recognizing that the human itself is fully material. So when we talk about the human and the non-human, the latter does not just mean other animate life forms such as those we encounter in the oceans, but also the basic building blocks or materials which form the rocks and undersea mountains, as well as the physical structures humans have created in order to explore them. Thus, for instance, the assemblage which is a new submersible, as will be seen later in the article, is itself determined by the materials out of which it is constructed-it is what makes certain activities possible and others impossible or less likely, and which enables humans to relate to the deep oceans in a way previously not possible. Hence assemblage theory is connected to what is called "the New Materialism" within philosophy, acknowledging the ways in which material reality shapes human responses, is also subject to human manipulation, and even more profoundly argues that what we call "the human" is itself always already fully part of that materiality and as much in process of change and becoming as the rest of the "material world."

A definition provided by one the originators of this approach which sounds very technical but is consistent with my own description is as follows:

What is an assemblage? It is a multiplicity which is made up of heterogeneous terms and which establishes liaisons, relations between them, across ages, sexes and reigns-different natures. Thus the assemblage's only unity is that of a co-functioning: it is a symbiosis, a "sympathy." It is never filiations which are important but alliances, alloys: these are not successions, lines of descent, but contagions, epidemics, the wind. (Deleuze \& Parnet, 2002, p. 69)

What this also makes clear is that the traditional way of thinking which places "things," both humans and non-humans, into some sort of hierarchy, is now no longer adequate. So it is not about "lines of descent" but rather an acknowledgement that, as another contributor to the theory suggests "all things equally exist, yet they do not exist equally" (Bogost, 2012, p. 11). Now this is to take engineers and marine biologists out into the finer detail of contemporary philosophy and a liminal space where they might feel "out of their depth." It could however be argued that one of the insights of the environmental movement is that the damage that humans have wrought upon the planet stems directly from our view of ourselves as somehow superior to the rest of creation, and thus in a position to exploit and manipulate "others" - sometimes humans but often non-humansfor our own interests and benefit. 
Another thinker from this philosophical approach talks about "vibrant matter" and offers the example of the North American blackout in August 2003 to describe the interconnections which often remain hidden. She gives a similar definition of assemblages:

Assemblages are ad hoc groupings of diverse elements, of vibrant materials of all sorts. Assemblages are living, throbbing confederations that are able to function despite the persistent presence of energies that confound them from within.... Assemblages are not governed by any central head; no one materiality or type of material has sufficient competence to determine consistently the trajectory or impact of the group. The effects generated by an assemblage are, rather, emergent properties, emergent in that their ability to make something happen is distinct from the sum of the vital force of each materiality considered alone. (Bennett, 2010, p. 24)

This approach is characteristic of the New Materialism and has links to specifically ethical and faithbased understandings (Crockett \& Reader, in press).

To set this out I refer to another of the major texts from this new source:

Thinking climate change requires thinking ecologically and thinking ecologically requires us to think how we are both embedded in a broader natural world and how non-human things have power and efficacy of their own. However, because we had either implicitly or explicitly chosen to reduce things to vehicles for human discursivity, it became impossible to theorize something like climate change because we only had culture as a category to work with. Having brought about the dissipation of the material in the fog of binary oppositions introduced by signs, there was no longer a place for thinking the real physical efficacy of fossil fuels, pollutants, automobiles, sunlight interacting with the albedo of the earth and so on. (Bryant, 2014, p. 4)

In response therefore, the task is to rethink the concept of society and indeed of humans themselves, and to propose that there is only nature, and that culture itself is a formation of nature. Within this there is a multiplicity of assemblages containing humans and non-humans, the latter also including the machines and technological devices that are the means of engineering contributing both positively and negatively to the environmental challenges collectively to be faced. The ethical task ahead is to identify which are the positive and constructive assemblages and which the damaging and sometimes deadly ones. We need to question in each specific instance what it is that a particular assemblage can do and to evaluate its contribution to the wellbeing of the whole.

\section{ENGINEERING}

Moving now to consider some engineering projects that attempt to address the issues above from the sphere of marine biology, and also climate change more generally, we remind ourselves of the questions to be asked. Who benefits from these-and this must include reference to both human and non-human-and what values are at work, either implicit or explicit? This will enable the critical mapping of the projects as specific assemblages and assist in determining the extent to which they are constructive or destructive.

A report in the journal Process Engineering on October 2nd 2014 recorded that the first commercial scale Carbon Capture and Storage (CCS) plant was to begin operating on that day in Canada (McKenna, 2014). The 110MW coal-fired power unit with amine capture technology is claimed to be able to capture around 1 million tons of $\mathrm{CO}^{2}$ each year, thus cutting the unit's emissions by $90 \%$. The facility has cost around $£ 751$ million to build. At the moment Europe lacks a pipeline of CCS projects and lags behind in terms of technological development, although there are two 
commercial-scale CCS projects being developed in the UK, at Drax and at Peterhead, under a government programme. Both would transport $\mathrm{CO}^{2}$ to the North Sea. This first project to come into full operation is being claimed as a major breakthrough in showing that brown coal can be used to generate electricity with only one quarter of the carbon emissions of natural gas and one tenth of the emissions caused by burning coal historically. So who benefits? What values are involved? What assemblages are to be identified? It is not easy to evaluate, but such technology and advanced engineering is not universally accepted as either being without faults, or necessarily a good way of tackling the challenges of climate change. The obvious immediate beneficiary will be the energy industry itself as it allows sections of it to continue operating when many would argue that coalfired power stations and their proliferation in places such as China, are the very units that should no longer be built and their expansion limited if not prevented altogether. It is not known as yet whether the impacts being claimed for this process can be delivered, therefore their benefit for the non-human in terms of taking pressure off the oceans and their capacity to absorb $\mathrm{CO}^{2}$ is a matter of conjecture. Clearly those directly involved in developing this technology will benefit financially. The other obvious aspect of this is the political one, and the extent to which governments supporting these developments as part of their overall energy policy, can claim to be mitigating the worst impacts of $\mathrm{CO}^{2}$ and climate change, when in fact their actions are already a matter of "too little too late." So is this an example of the use of engineering to provide window dressing for the politicians and their allies in the energy industry rather than a genuine attempt to address the wider problems? This particular assemblage of engineers, the physical products and components required for the plants themselves, the external funding sometimes government-based and, if not, privately resourced, the coal and its methods of extraction and burning, and then the $\mathrm{CO}^{2}$ in whatever form it is then processed and dealt with, cannot yet be said to be unambiguously of benefit for the human or the non-human. The values would appear to be those of the established capitalist structures which still see the non-human as resources to be manipulated and exploited for the benefit of the few and to the detriment of the many, if indeed it is the case that this fails to stem the rising emissions of $\mathrm{CO}^{2}$ globally.

Another less than obviously benign engineering innovation currently under development is to be found in the oil industry. In the light of the massive BP oil spill in the Gulf of Mexico a few years ago, the impact of this arm of the energy industry is also under scrutiny. The state funded and controlled company Statoil in Norway, is developing a series of underwater plants connected by pipelines that can operate effectively at a deeper level than previously possible. This again, it is argued, will enable normal extraction processes to continue whilst limiting environmental impacts and damage. Without state funding no company could afford to take the risks of developing this form of technology and the engineering expertise required. So very much as with the CCS plant, this is a project which makes claims that are yet to be substantiated in practice; which enables the energy industry to continue some form of business as usual; which encourages rather than challenges dependence upon non-renewable sources of energy, and which appears to support the assemblages of corporate interests, government policies and the financial concerns of those developing the technology and implementing it. Whether this particular development within the field of Sub Sea Oil and Gas is really of benefit to the wider planet then is a matter of debate to say the least, but it will no doubt be presented to the public as another means by which human ingenuity and investment is being employed to counter the effects of environmental damage, whilst allowing corporate interests to continue unchallenged. Hence media and public relations coverage is another aspect of this type of assemblage and needs to be viewed carefully and critically.

Related to both of the above is of course the plastics industry, the materials being derived from oil and gas, and both a source taken for granted in our culture and also a major component of human waste. Huge amounts of plastic waste end up in the oceans, flowing down rivers, sewers and storm 
drains. Boats are another source of plastic pollution with materials being dumped overboard and fishing boats discarding large amounts of fishing gear. One of the problems with this is that plastics take a long time to degrade, hence the oceans are subject to an ever increasing level of plastic debris both floating on the surface and also sinking to the seabed. Similarly, plastic debris on shorelines is also a problem. An example of the levels of ocean-going plastic was a study collecting plastic in the North Pacific gyre which revealed an average of 334,271 pieces of the material per square kilometer (Mladenov, 2013, p. 58). This is harmful to many species of marine life which either become entangled in it or ingest it. Seabirds and sea turtles are particularly at risk. Although some measures have been put in place to agree to limit the levels of disposal these are difficult to monitor and enforce. The obvious response to this would be to develop and encourage more means of recycling the waste on land before it ever reaches the oceans, and there are indeed resources for this from within materials science engineering which need to be more widely disseminated and implemented. However, this is yet another assemblage which involves politics, the media, the lifestyle of ordinary households, as well as the engineers who develop the technology, and of course the physical materials themselves and their potentials for change over time. At the moment this particular assemblage is detrimental to the wider planet but it is one that could be turned around to become of benefit.

A development that is perhaps more positive is that of submersibles mentioned earlier and which are now able to take humans down to ocean depths previously not possible. Triton small submarines or submersibles are now available as a result of engineering advances and are being used for the purposes of deep sea exploration. The irony however, is that these were originally developed as a purely commercial product, and the latest accessory for those millionaires who own sea-going yachts and who wanted an "experience" that only the most wealthy could enjoy. As with so many of the assemblages we have identified, there is an ambiguity about the process and the impact of the engineering technology so that there are benefits for the few which can sometimes be turned around to the benefit of the many, but only when sufficient funds or political will is available to make this possible. Why are these submersibles of value for marine biology? The deep ocean has been believed to be a dark and lifeless environment, although it is the largest ecosystem on the planet, covering $80 \%$ of the entire global ocean and $79 \%$ of the planet's habitable living space. Yet, until recently, it has been largely inaccessible to humans. There is little light, extreme pressure and low temperatures and yet there is a great diversity of marine life present. Up until now only about $5 \%$ of the deep ocean bottom has been explored using remotely operated robotic vehicles and less than about $0.01 \%$ has been surveyed in any detail (Mladenov, 2013, p. 105). Now it has become possible for humans to go down into the depths in safety through the development of submersibles, a whole new world has opened up and we can begin to learn more about the life forms which are an integral part of the lives we share on the planet. The questions this raise however are whether we will see this as yet another "resource" to be exploited for human benefit, or whether instead this ecosystem will be valued in its own right and its integrity and existence respected. Once again then, it all depends on whether or not humans can change their understanding of the relationship between nature and culture, and grasp that culture itself is simply another component in the greater assemblage that is the world of which we are all a part, and over which we do not and cannot exercise some sort of proprietorial control. Philosophy, politics, marine biology and engineering form together the combination of disciplines which will shape the human response to the non-human ecosystem of the deep ocean.

In summary then, it has been shown that assemblage theory provides a means of analyzing engineering developments and innovations in a way that reveals the interconnected and complex 
nature of the interactions between the human and the non-human, those including more than just other animate creatures, but also the material components which are both shaped by and shape those interactions. Rather than presupposing a hierarchy of beings with humans at the summit and everything else demoted to being a resource to be manipulated or exploited, this flat ontology assumes a much more level playing field within which humans are not lone autonomous individuals, an understanding of which is seen to have been so damaging to the planet, not least as perceived through marine biology. Thus it also suggests a means of making ethical evaluations of such engineering advances based on whose interests and needs are being served. Each assemblage must be examined in detail in order to assess whether it is damaging to the whole or of benefit to it, and only then can alternatives be proposed and developed.

\section{ACKNOWLEDGEMENTS}

Thanks also to Drs. Charles and Anne Sheppard from the University of Warwick, UK for their help and guidance on the subject of Marine Biology, Coral Reefs and Marine Protection Areas (Hope Spots) in particular.

\section{REFERENCES}

Baillie, C., Kabo, J. \& Reader, J. (2013). Heterotopia: Alternative pathways to social justice. Winchester, UK: Zero Books.

Bennett, J. (2010). Vibrant matter: A political ecology of things. Durham, NC: Duke University Press.

Bogost, I. (2012). Alien phenomenology, or, what it's like to be a thing. Minneapolis: University of Minnesota Press.

Bryant, L. R. (2014). Onto-cartography: An ontology of machines and media. Edinburgh, UK: Edinburgh University Press.

Crockett, C. \& Reader, J. (in press). Ecology and social movements: The new materialism and relational Christian realism. [Forthcoming chapter in the Palgrave Macmillan Radical Theology Series].

Deleuze, G. \& Parnet, C. (2002). Dialogues II. New York, NY: Columbia University Press.

McKenna, J. (October 2, 2014). World's first CCS plant opens. Process Engineering. Retrieved from http://processengineering.theengineer.co.uk.

Mladenov, P. V. (2013). Marine biology: A very short introduction, Oxford. UK: Oxford University Press. 